\title{
Le sport et les seniors : des pratiques spécifiques ?
}

Fabrice BURLOT, Brice LEFÈVRE, Laboratoire de sociologie de l'INSEP.

Article paru dans : RETRAITE ET SOCIÉTÉ (ISSN 1167-4687), $n^{\circ} 58$, novembre 2009

Les 14 tableaux de données ne sont pas reproduits. Pour les consulter voir l'article original.

En France, le poids des activités physiques et sportives chez les seniors a progressé depuis plus de vingt ans. En 1985, $60 \%$ des personnes de plus de 55 ans déclaraient en pratiquer (Irlinger, Louveau, Metoudi, 1987). L'enquête 2000 en dénombre 73 \% (Mignon, Truchot, 2000), chiffre qui pourrait encore augmenter. Cette situation est la conséquence de plusieurs phénomènes. En premier lieu, le pourcentage croissant des plus âgés dans la population française (Desplanques, 2005; Richet-Mastain, 2006): l'espérance de vie dépasse aujourd'hui 75 ans pour les hommes et 80 ans pour les femmes (Pinson, 2006). En outre, depuis 2005, la génération du «papy-boom » arrive à l'âge de la retraite (Rochefort, 2000). Si cette évolution se confirme, les plus de 60 ans représenteront plus de $26 \%$ de la population française en 2020, alors qu'ils n'en représentaient que $19 \%$ en 1990 et à peine $10 \%$ en 1900 (Insee, 1990). En second lieu, ils bénéficient d'un temps libre important puisque la plupart sont aujourd'hui à la retraite. En effet, l'âge de la retraite est progressivement passé à 60 ans, mouvement accentué par des départs en préretraite favorisés par les entreprises (Gaultier, 2003). Enfin, les retraités d'aujourd'hui ont réellement une culture sportive. Ils font souvent partie de la génération « sport », c'est- à-dire des Français du «baby-boom » qui se sont largement engagés dans les fédérations sportives à partir des années 1960 et qui ont entrainé une progression rapide du nombre de licenciés qui, entre 1960 et 1985, est passé de 3 à 12 millions (Pociello, 1999). Ces nouveaux retraités considèrent le sport comme un facteur de santé, — de retardement du vieillissement et d'aide pour se maintenir dans les canons esthétiques de la jeunesse, comme le souligne Feillet (2000) - , mais aussi comme un élément de socialisation et, dans ce sens, de protection contre le vide social que peut entraîner le passage dans le monde des « inactifs » (Pichot, 2002).

Ces constats appellent une quantification des liens qui unissent les seniors et les pratiques physiques et sportives, et laissent en suspens certaines questions: qui pratique? Dans quelles conditions? Pourquoi ?

On s'interrogera ainsi dans un premier temps sur les caractéristiques sociodémographiques des seniors engagés dans des activités physiques et sportives: les hommes et les femmes s'impliquent-ils de la même manière? La pratique est-elle liée à l'origine sociale? Les retraités pratiquent-ils davantage que les non-retraités?

On étudiera ensuite la nature et les formes de pratique propres aux seniors.

S'inscrivent-elles dans la continuité des activités des plus jeunes ou traduisent-elles une rupture? Entraînent-elles une redéfinition du paysage sportif et peut-on observer des évolutions des modalités de pratique? Qu'en est-il de l'opposition entre les pratiques hors institutions et institutionnalisées? 
On s'interrogera enfin sur les motivations qui poussent les seniors à l'exercice sportif. Sont-elles semblables à celles des plus jeunes ou peut-on mettre en évidence des spécificités?

\section{Seniors et pratiques sportives}

Dans les pratiques sportives en général, le terme senior ${ }^{1}$ fait référence à la catégorie adulte, les sportifs âgés de plus de 50 ans relevant de celle des vétérans. Nous attirons l'attention du lecteur sur le fait que ce n'est pas à la définition sportive des seniors que nous ferons référence dans ce texte mais à la catégorisation sociale des personnes de 50 ans et plus, notamment utilisée dans les études du Credoc.

Dans notre société, l'arrêt de l'activité professionnelle et donc, souvent, l'âge de la retraite marquent le point de rupture entre le monde des plus jeunes et celui des «vieux ». Or, depuis la seconde moitié du vingtième siècle, cet âge n'a cessé de se réduire, passant de 65 ans à 60 ans et parfois, dans certaines conditions de mise en préretraite, sous le seuil des 55 ans. Si l'âge qu'il convient d'associer au mot «senior » ne fait pas l'unanimité. Il n'en reste pas moins qu'une partie des 50-60 ans est aujourd'hui concernée par les problématiques qui étaient hier l'apanage des plus de 60 ans. Soit parce qu'elles sont effectivement en retraite, soit parce qu'elles pratiquent unsport dans lequel elles ont le statut de vétéran et que, de façon générale, elles se situent à la limite de la vie active et de la vie inactive. Aussi, nous avons décidé de ne pas nous limiter aux personnes de plus de 60 ans et d'étendre notre travail à une connaissance plus précise des comportements sportifs des seniors de 50 ans et plus.

Si les 50-59 ans représentent une classe d'âge spécifique, reste posée la question de l'homogénéité de la population des plus de 60 ans. Pour qualifier celle-ci, la presse et le monde économique utilisent deux notions: le troisième âge, qui s'applique à des retraités actifs, et le quatrième âge, ou « grand âge », qui concerne les retraités plus âgés et beaucoup moins actifs. Cependant, cette distinction ne repose pas vraiment sur une question d'âge mais interroge davantage l'idée d'être vieux. En 1950, on était vieux à 65 ans. En 2040, on pourrait ne l'être qu'à 82. Ce recul de l'âge de la vieillesse rend compte certes d'un gain d'espérance de vie mais entraîne surtout un allongement du temps actif (Rochefort, 2000) qui laisse augurer, pour l'avenir, un développement accru des activités consommées par le troisième âge avant l'entrée dans le quatrième (Follea, 1997). La pratique sportive est à ce titre un excellent champ, qui permet d'appréhender les transformations des pratiques avec l'avancée en âge (Henaff-Pineau, 2008).

Mais avant d'entrer dans le vif du sujet, il nous faut également définir ce que nous entendons ici par sport. On peut aujourd'hui considérer le sport comme un fait social total (Pociello, 1999) tant le phénomène prend de l'ampleur et traverse les différentes composantes de la société française. Pourtant, malgré — ou peut-être en raison — de cet intérêt, l'acception du terme « sport » reste sujet à polémique compte tenu de sa polysémie. «Faire du sport, c'est faire de la compétition » affirment certains pratiquants. «Pour moi, faire du sport, c'est marcher le long du canal » dira un retraité. Des controverses existent également chez les scientifiques ${ }^{2}$ qui, devant définir un objet lié à un univers social, en limitent les frontières pour l'étudier. Les débats sont d'autant plus nourris que le paysage des pratiques sportives connaît des transformations multiples: massification, diversification des formes de pratique, émergence d'activités nouvelles, importance du sport auto-organisé, et surtout avènement du sport comme véritable norme sociale.

Lorsqu'on étudie les pratiques sportives des Français sous l'angle démographique, engageant ainsi une mesure de la pratique, surgissent plusieurs interrogations: quels types de pratiques et quels 
types de pratiquants choisir? Doit-on intégrer les activités d'entretien physique? Doit-on uniquement prendre en compte les pratiques institutionnalisées? Et comment peut-on considérer celles des retraités?

Sur cette question complexe, le laboratoire de sociologie du sport de l'Insep, chargé en 1985 de mesurer les pratiques sportives des Français, a rompu avec les conceptions antérieures. Son enquête fut une véritable innovation car toutes les précédentes s'appuyaient sur des définitions restrictives (Louveau, 2002), souvent liées aux licenciés et à l'inscription à un club, renvoyant donc uniquement à la compétition. Plutôt que d'imposer une définition a priori, les sociologues de l'Insep ont laissé les enquêtés définir eux-mêmes ce qu'ils entendaient par «faire du sport » et ont opté de facto pour une conception large: «le sport, c'est ce que font les gens quand ils pensent qu'ils font du sport» (Irlinger, Louveau, Métoudi, 1987). La stratégie était de renseigner toutes les pratiques renvoyant à l'idée de faire du sport », d'intégrer un ensemble de pratiques de loisirs liées au plein air ou à la ville ou bien encore à l'entretien du corps. Cela permettait ensuite de discerner, à partir des déclarations des enquêtés, les modalités de leurs pratiques: intensité, lieux, moments, cadre institutionnel ou non.

\section{Aspects méthodologiques de l'enquête 2000}

Cette enquête a pour objectif de décrire et de mesurer l'ensemble du phénomène sportif en France. Pour répondre techniquement à cet objectif, deux questions préalables ont été posées:

Q1 : " Au cours des 12 derniers mois, avez-vous fait, même occasionnellement ou même pendant vos vacances, des activités physiques et sportives, ou d'autres activités de ce type, en dehors des cours obligatoires d'éducation physique et sportive à l'école ? »

Q2: «Pouvez-vous citer le plus précisément possible toutes les activités physiques et sportives que vous avez pratiquées au cours des douze derniers mois?»

À la suite de ces deux questions, une relance a été effectuée ${ }^{3}$.

On remarque ici l'importance des termes utilisés dans le questionnement: les mots « activités physiques et sportives » permettent d'englober un large éventail de pratiques de libre choix, excluant explicitement les activités physiques et sportives scolaires à caractère obligatoire.

Le moyen choisi pour recueillir les données est l'enquête par questionnaire à une échelle nationale. Un échantillon de 6526 personnes (méthode des quotas), représentatif de la population des Français de 15 à 75 ans (métropole + Dom-Tom), a été prélevé en juillet 2000. Par téléphone, chaque individu a répondu à un questionnaire reprenant le type de questionnement présenté en introduction. En considérant les données du recensement national, les 6526 personnes interrogées correspondent à 44,1 millions de personnes ( 1 individu de l'échantillon = 6761 individus dans la réalité).

Dans l'évaluation globale de la situation sociale et culturelle, une variable construite « indicateur de richesse » et une variable « diplôme » ont été privilégiées. «L'indicateur de richesse » a été créé en faisant le rapport du salaire du foyer au nombre de personnes composant le foyer. La variable « profession » n'a pas été traitée, compte tenu notamment de l'émiettement des données de l'échantillon, provoqué par le nombre important de modalités de l'âge et de la profession, fragilisant notoirement les résultats. Deux questions concernant l'âge et le sexe ont également été utilisées.

Dans cette étude, nous utilisons seulement les questions suivantes, relatives à l'engagement sportif (fréquence, période de pratique, appartenance à une association ou à un club, licence fédérale) et à la motivation : 
« Pour chaque activité physique et sportive que vous pratiquez, pouvez- vous maintenant me dire quand et à quelle fréquence vous la pratiquez ?»

Q4 - «Quand pratique-t-on?

« Pendant l'année, y compris pendant les périodes de vacances », « En période de vacances seulement », «En dehors des périodes de vacances seulement ».

Q4b - « À quelle fréquence? »

«Moins d'une fois par mois », « 1 à 2 fois par mois », « 1 fois par semaine »,

« $\quad 2$ à 3 fois par semaine », «plus de 3 fois par semaine », « pendant un période donnée/une saison ».

Q5 - « Êtes-vous membre d'un club ou d'une association sportive? »

Q5ter - «Concernant la pratique $\mathrm{n}^{\circ} 1$, êtes-vous titulaire d'une licence délivrée par une fédération sportive?»

Q6 - « Et diriez-vous plutôt qu'en pratiquant cette activité, vous recherchez :

« le risque », « la gagne, la compétition, la performance », « les sensations », «l'amélioration de vos performances », « la rencontre avec les autres », « la santé », « le contact avec la nature », « être bien dans votre peau », « la détente », « l'engagement physique », « la dépense physique », « le plaisir d'être avec ses proches ».

Pour l'analyse des données sociodémographiques, les pourcentages en lignes sont fournis pour les croisements de deux variables. En ce qui concerne le croisement des différents types de pratiques physiques et sportives avec l'âge, on a effectué un test exact de Fisher pour identifier une éventuelle relation entre deux modalités. Si le résultat est significatif, on a calculé le «pourcentage de l'écart maximal à l'indépendance » (PEM, Cibois, 1993) pour préciser le sens et l'intensité de la relation. Ce PEM varie de $-100 \%$ pour une répulsion totale à $+100 \%$ pour une attraction totale, ce qui permet une hiérarchisation globale des relations identifiées. Le traitement des données est réalisé avec un programme d'analyse de tableaux de contingence sur le logiciel R.

Quant à la distribution de l'âge (tableau 1), les moins de 50 ans représentent environ les deux-tiers des Français âgés de 15 à 75 ans et les seniors correspondent à un tiers de l'effectif. De 50 à 75 ans, les parts des tranches d'âge d'amplitude constante (cinq années) sont comparables, oscillant entre 6 et $8 \%{ }^{4}$.

\section{Les caractéristiques sociodémographiques des seniors et leur engagement sportif}

Les pratiques physiques et sportives des seniors semblent marquées par deux paliers temporels. Le premier, à 50 ans, conforte l'option de traiter ensemble les plus de 50 ans; le second, à 70 ans, montre une véritable distinction entre les pratiques sportives des seniors. Après 50 ans, la nonpratique devient en effet plus importante, demeurant assez stable jusqu'à 69 ans, pour augmenter considérablement à partir de 70 ans (tableau 2, page 140). De 30 à 49 ans, les non-pratiquants représentent entre $14 \%$ et $16 \%$ de la population française contre $21 \%$ à $24 \%$ entre 50 et 69 ans et près de $38 \%$ de 70 à 75 ans. 
Analysés séparément, les hommes et les femmes connaissent des situations différentes et pourtant comparables (tableau 4, page 141). Les femmes pratiquent certes moins que les hommes, mais les deux sexes connaissent entre 50 et 69 ans chacun un pic de pratique: pour les femmes entre 55 et 59 ans, et pour les hommes entre 65 et 69 ans ${ }^{5}$. Chez les hommes, ce pic d'activité s'intercale à l'intérieur d'une période d'effritement régulier du taux de pratique depuis le plus jeune âge (exception faite de la période de 40 à 49 ans). Chez les femmes, il fait suite à une baisse considérable de la pratique qui s'engage à partir de 45 ans: $15 \%$ des femmes âgées de 40 à 44 ans ne pratiquent pas contre $29 \%$ de 50 à 54 ans.

Par ailleurs, non seulement le taux d'activité diminue avec l'âge, confirmant un processus progressif de désengagement ou de « déprise » couramment décrit (Barthe et al., 1988; Caradec, 2001), mais le sentiment d'être sportif s'atténue également. Les déclarations spontanées de pratique sont beaucoup moins nombreuses après 50 ans. Plus nombreux sont ceux qui se déclarent «sportifs » après relance. Le phénomène est plus accentué chez les femmes et devient même particulièrement important pour celles de 70 ans à 75 ans. Pour ces dernières, les déclarations de pratique après relance sont alors supérieures aux déclarations spontanées. Cette situation explique les différences avec l'enquête Insee 2003 sur le même sujet (Mignon, Truchot, 2005): le choix de questions fermées sans relance réduit considérablement le taux de pratique, notamment chez les personnes les plus âgées et chez les femmes. On trouve ainsi dans l'enquête 2000, $41 \%$ de femmes entre 70 et 75 ans se déclarant non pratiquantes, contre plus de $64 \%$ dans l'enquête 2003. Celle-ci ne permet pas de mesurer un certain nombre d'activités informelles que les seniors femmes ne considèrent pas «a priori » comme des pratiques sportives mais qui constituent néanmoins des formes d'exercice physique. Notamment, « faire de la marche » ne renvoie pas à la même symbolique que le terme « randonnée pédestre » utilisé dans l'enquête 2003 pour caractériser les activités de marche dans une question fermée.

Enfin, le niveau de vie des Français comme leur niveau scolaire agissent fortement sur l'engagement sportif des seniors, surtout pour les plus âgés : $56 \%$ des 70-75 ans titulaires de revenus inférieurs ne déclarent aucune pratique contre seulement $28 \%$ des titulaires de revenus supérieurs (tableau 5 , page 143). L'écart entre les riches et les moins riches s'accentue avec l'âge: il est en moyenne de 9 points pour les moins de 50 ans, avoisine les 15 points entre 50 et 69 ans et passe à 28 points pour les 70-75 ans. De plus, chez les plus riches, la non-pratique progresse lentement mais sans interruption jusqu'à 59 ans, alors que pour les moins riches, même si on perçoit quelques écarts, elle connaît une certaine forme de stabilité jusqu'à 49 ans, et un véritable point de rupture à 50 ans. De la même façon, le niveau scolaire joue sur l'engagement sportif. Les seniors les plus diplômés pratiquent davantage et, contrairement à toutes les sous-populations de seniors étudiées, leur taux de pratique progresse globalement avec l'âge (tableau 6, page 144). À partir de 60 ans, leur pratique sportive augmente nettement, alors que chez les moins diplômés, la non-pratique progresse par paliers successifs, environ $18 \%$ de 20 à 49 ans, entre 20 et $26 \%$ jusqu'à 69 ans et $43 \%$ à partir de 70 ans.

Pour conclure, les seniors de 50 à 69 ans n'apparaissent pas comme une population particulièrement spécifique du point de vue socioculturel. Dans la continuité des autres tranches d'âge, on note les mêmes distinctions : le sexe, les diplômes et la richesse restent des facteurs déterminants de la pratique. En revanche, si l'entrée dans cette catégorie d'âge laisse apparaître une augmentation de la non-pratique, quelles que soient les catégories de personnes (en dehors du cas atypique des plus diplômés), on ne note pas de rupture radicale de pratique. Elle se caractérise plutôt par une stabilité prolongeant un effritement progressif de la pratique, dont l'origine se situe avant 50 ans et à 
l'intérieur de laquelle on note presque systématiquement un point plus ou moins marqué de rupture temporaire : une mise en suspens du phénomène 144 d'effritement, voire un sursaut de pratique. Pour les femmes, les moins riches ${ }^{6}$ et les non-bacheliers, ce sursaut concerne les 55-59 ans et pour les hommes et les riches, plutôt les 65-69 ans.

Cette stabilité et ce sursaut différencié de pratique nourrissent des interrogations et soulignent la complexité du phénomène. D'un côté, le temps libre dégagé par la retraite ne semble apparemment pas entraîner une mise en cause radicale de la situation sportive des individus puisque les taux de pratique évoluent peu. On pourrait presque penser que la retraite ne modifie en rien leurs réponses aux questions relatives à la pratique d'un sport. D'un autre côté, il faut nuancer cette conclusion en rappelant que cette stagnation succède à une baisse régulière de la pratique et révèle plutôt l'existence d'une situation de rupture par rapport à la situation des moins de 50 ans. Mais alors pourquoi constate-t-on simplement une stagnation et pas une augmentation, d'autant que l'enquête met en évidence une différence significative entre l'engagement sportif des retraités et des nonretraités, les retraités faisant davantage de sport que les non-retraités? (tableau 2, page 140).

En réalité, cette période de stabilité chez les seniors semble s'expliquer par une compensation entre les retraités sportifs, dont la part augmente, et les non-retraités sportifs, dont le nombre diminue. Si on compare les déclarations de pratique sportive des retraités et des non-retraités dans le temps (tableau 3, page 141), on constate que la non-pratique sportive s'accroît considérablement chez les non-retraités, passant de $22 \%$ pour les 55-59 ans à $73 \%$ pour les $70-75$ ans. Elle prolonge l'effritement constaté avant 50 ans. En revanche, chez les retraités, la pratique augmente légèrement de 55 à 69 ans, passant de $18 \%$ à $21 \%$ pour véritablement s'intensifier à partir de 70 ans. En conséquence, même si, a priori, la retraite joue indéniablement sur la pratique sportive, on peut néanmoins s'interroger sur la nature de son influence avec cette mise en suspens de l'effritement. Quelques questions demeurent ainsi posées. Les sportifs continuent-ils massivement leurs activités sportives ? Quelle est la part des retraités nouvellement sportifs? Et, pour aller directement au coeur du sujet: la retraite rend-elle les Français plus sportifs?

Évidemment, seule une analyse longitudinale pourrait nous permettre de répondre à cette question. En effet, les sursauts observés dans les sous- populations, qu'il serait possible de considérer comme les conséquences de la situation de retraite, ne correspondent pas en réalité aux périodes d'accès massif à la retraite. Ceux-ci se situent en dehors de la période centrale des 60-64 ans, dans laquelle $70 \%$ des Français sont à la retraite (tableau 2, page 140). C'est pendant la période où les hommes et les femmes passent massivement à la retraite (Desplanques, 2005) que les taux de pratique sont les moins élevés comparés à ceux des autres tranches d'âge.

Ce décalage surprend et même si notre méthode ne permet ni de prendre en compte l'effet de génération certainement sensible, ni de formuler des réponses précises, elle ouvre néanmoins quelques pistes de réflexion.

La retraite s'inscrit dans une période de prise de conscience du vieillissement corporel et de résistance à ses effets. Elle joue le rôle d'un marqueur temporel délimitant le monde des actifs et le monde des inactifs, et modifie le rapport au sport, comme le souligne Feillet (2000) à propos de retraités ruraux. Certes, aujourd'hui, le passage à la retraite n'est plus uniquement considéré comme un traumatisme. Il est également perçu comme une situation espérée et préparée. Mais le nouveau temps libre n'est peut-être pas systématiquement occupé par le loisir sportif, surtout pour les nonsportifs. Pour cela, il est nécessaire que le retraité en éprouve immédiatement le besoin ou lui trouve une fonction. On comprendrait difficilement que les sportifs, sauf problèmes de santé, rompent 
brutalement avec leurs habitudes au moment de leur retraite. Les travaux de Henaff $\neg$ Pineau (2008) montrent au contraire que la retraite marque une phase d'amplification des pratiques physiques et « constitue une étape privilégiée d'engagement ou de ré-engagement sportif ». Pour les retraités nonsportifs, on peut imaginer la possibilité d'un engagement différé. Cette hypothèse rejoindrait l'idée selon laquelle une partie des retraités, les hommes surtout, ne s'engageraient pas immédiatement dans des activités nouvelles, préférant mener d'abord à bien leurs tâches domestiques personnelles, « comme s'il fallait que les travaux soient achevés pour se sentir vraiment à la retraite et pouvoir se consacrer à autre chose » (Caradec, 2004, p. 51). Si l'on peut donc oser quelques conjectures sur le décalage perceptible chez les 65-69 ans en évoquant un report différé de pratique, au moins pour les «nouveaux » sportifs, quelles raisons trouver au pic de pratique des 55-59 ans, alors même que de nombreux Français ne sont pas encore à la retraite?

Si la période entre 50 et 69 ans laisse en suspens un certain nombre de questions, la stabilisation apparente du niveau d'activité sportive masque des mouvements de population complexes qui ne manquent pas d'affecter également les modalités de pratiques.

Les seniors pratiquent moins, mais aussi différemment. Avec l'âge, ils aspirent à des activités de moins en moins institutionnalisées. Ils sont moins nombreux à être licenciés, membres d'un club ou d'une association (tableau 2, page 140), ce qui explique peut-être également leur taux de réponse spontanée peu élevé. Sils se considèrent moins sportifs que les plus jeunes, c'est surtout parce que le sport renvoie pour eux à une pratique d'engagement. La marche n'est pas toujours spontanément considérée comme une pratique sportive, surtout pour un ancien compétiteur. Au contraire, se livrer à une activité dans un club s'inscrit immédiatement dans un schéma de pratique sportive. Cette réalité permet d'ailleurs de nuancer l'idée selon laquelle les retraités rechercheraient dans le monde associatif une nouvelle sociabilité compensant la perte de leurs relations professionnelles. On note en effet un rebond aux alentours de 60 ans, mais les taux restent bien en dessous de l'engagement institutionnel sportif en général. Surtout, la part des retraités parmi ces sportifs institutionnels n'est pas connue (les effectifs dans l'enquête 2000 sont insuffisants pour obtenir des résultats fiables).

Ce désengagement institutionnel des seniors diffère selon le sexe, le niveau de revenus et de diplômes (tableau 3, page 141). Certes, tous connaissent la rupture vers 70 ans. Mais si le taux d'institutionnalisation des pratiques masculines est stable entre 50 et 69 ans, il augmente légèrement chez les femmes entre 50 et 64 ans. S'ensuit une baisse jusqu'à 75 ans, alors que les hommes se signalent par un rebond, davantage marqué à partir de 60 ans. Pour les plus riches et les plus diplômés, l'engagement institutionnel se distingue également, au cours de la période de 50 à 69 ans, par des taux d'adhésion beaucoup plus importants que pour les moins riches et les moins diplômés.

\section{Les activités et les modalités de pratique des seniors : entre homogénéité et hétérogénéité}

Avec l'âge, les sportifs limitent leur portefeuille d'activités (Truchot, 2002), tout en s'engageant davantage dans les activités qu'ils conservent (tableaux 7, 8 et 9, ci-dessous et page 148). En outre, ils se concentrent sur des activités physiquement moins dures (tableau 10, page 149). Cette évolution confirme l'idée de stratégies de substitution et d'adaptation liées au processus de « déprise »(Caradec, 2004). Assez paradoxalement, plus on est vieux et plus la fréquence de pratique est élevée: $57 \%$ des sportifs de plus de 60 ans déclarent pratiquer au moins deux fois par semaine, contre $42 \%$ pour les moins de 50 ans (tableau 7). 
Ainsi, les seniors consacrent davantage de temps que les plus jeunes à une seule activité. La monopratique est en effet caractéristique des plus de 50 ans (tableau 9): $44 \%$ des plus de 50 ans déclarent une seule activité, contre seulement $22 \%$ chez les moins de 50 ans. À partir de 70 ans, la tendance s'accentue encore avec $54 \%$ des 70-75 ans. Cette tendance pourrait évidemment être associée aux besoins de sociabilité, de sécurité et de santé, qui restent des motivations dominantes pour les plus de 50 ans (tableau 14, page 152). Se consacrer à une seule activité pourrait alors permettre à la fois de rester intégré à un tissu social associatif, de conserver un niveau d'activité permettant la préservation de la santé, et surtout de limiter les risques liés à l'exercice d'un nouveau sport. La pratique «essayiste » se trouve progressivement limitée. Ainsi, si les vacances sont souvent considérées par les moins de 50 ans comme une opportunité d'exercer des activités incompatibles avec le temps du travail, les pratiques ponctuelles diminuent avec l'âge. Seulement 28 $\%$ des 70-75 ans déclarent une pratique en dehors des vacances (contre $40 \%$ pour les moins de 50 ans) et $29 \%$ pendant les vacances (contre $55 \%$ ). Les seniors recherchent donc une pratique régulière (tableau 8 ).

S'agissant des pratiques privilégiées par les seniors, et afin de ne proposer que des tableaux de dimensions raisonnables, ceux-ci mentionnent uniquement les pratiques qui sont liées de façon significative à certaines tranches d'âge.

Sur les 70 pratiques croisées pour lesquelles l'effectif est supérieur ou égal à 30 individus, 50 sont en attraction ou en répulsion avec le fait d'être un senior, à un seuil de confiance de $95 \%$. Les seniors sont surreprésentés dans seulement 11 pratiques, alors qu'ils sont sous- représentés dans 39. Cette répartition déséquilibrée des attractions et des répulsions s'explique principalement par la forte multipratique des plus jeunes, très nombreux dans de multiples activités. Reste que la méthode du questionnaire ne permet pas d'analyser précisément le contexte de pratique et de la façon de pratiquer même si, dans le cas présent, les déclarations brutes sont prises en compte. Elles autorisent une étude plus fine des pratiques, avec par exemple des déclarations « marche loisir » qui sont discriminantes.

Les activités préférées par les seniors (tableau 10) appartiennent aux domaines de l'entretien du corps et du bien-être avec des affinités fortes (PEM à plus de $30 \%$ ) et des taux de pratiquants avoisinant $50 \%$ — la part des seniors dans la population est de 30 'Yo — pour le yoga, la marche (dont la marche « de loisir ») et le vélo d'intérieur. Sont également remarquables les surdéclarations des formes de pratiques les plus douces: gymnastique volontaire et gymnastique d'entretien, en opposition à la gymnastique sportive, ski de fond en opposition au ski alpin, et danse de salon en opposition au rock.

On retrouve dans ces quelques résultats une désaffection pour les activités de dépassement comme les pratiques fortement dispendieuses en énergie, compétitives et à risque. L'analyse de la hiérarchie des répulsions le confirme (tableau 11). Pour décrire les pratiques des seniors, il faut notamment prendre en compte leur faible participation ou leur quasi-absence dans certaines activités. Ces sousreprésentations sont particulièrement fortes à la lecture des PEM, tous inférieurs à $-81 \%$.

Si l'on change d'échelle et que l'on ne s'intéresse qu'à la sous-population des seniors en la découpant en deux classes d'âge comparables en volume, « de 50 à 59 ans » et « de 60 à 75 ans » (tableau 12, page 151), on retrouve pour partie les grandes différences relevées entre les seniors et les plus jeunes, à l'exception de la dimension compétitive fédérale. Chez les personnes âgées de 50 à 59 ans, on note des affinités avec des activités plus risquées, comme le VTT et le ski alpin, et des activités 
physiques soutenues, comme le footing/jogging ou la musculation, avec des PEM non négligeables. Seules les pratiques de marche ( «marche » et surtout «marche de loisir ») sont surinvesties par les personnes âgées de 60 à 75 ans. Le découpage en cinq classes d'âge (tableau 13, page 151) permet d'affiner l'analyse en mettant en évidence des pratiques déjà observées chez les moins de 60 ans, auxquelles s'ajoute la natation de loisir. Pour les plus âgés sont confirmées quelques tendances avec des PEM peu marqués. Chez les 55 à 59 ans, existe une préférence pour la baignade, et chez les 60 à 64 ans une prédilection pour le vélo de loisir. Le goût des plus âgés (70 à 75 ans) pour la marche se signale une nouvelle fois.

L'implication des seniors se caractérise certes par un amoindrissement $\mathrm{du}$ portefeuille de pratiques, mais aussi par une fréquence d'activité plus soutenue. Globalement, ils s'orientent vers des activités centrées sur le corps ou le bien-être et vers des modalités plus douces. On notera néanmoins une propension des 50-69 ans à la recherche de disciplines risquées et à forte dépense énergétique. Ces pratiques et ces désaffections s'éclairent par l'étude des motivations.

\section{Les motivations}

Les seniors préfèrent des activités à dimension hygiéniste, favorisant la santé physique et psychologique (tableau 14). Ils définissent le sport comme un moyen de résister au vieillissement et de prendre conscience que le corps est encore susceptible d'être actif (Feillet, Roncin, 2006).

À l'appui de cette idée se trouvent d'abord des données objectives tels les travaux montrant en quoi l'activité physique et sportive participe à la lutte contre l'ostéoporose et le diabète (Barello et al., 2002), à l'amélioration de la circulation et à la réduction des maladies cardiovasculaires (Faurobert, 1976; Dejardin et al., 1999). Ces effets bénéfiques sont soulignés par une série d'ouvrages plus ou moins scientifiques dans lesquels on enseigne l'art et la manière de mieux vieillir (Dessein, 2005; Longueville, 1978) et dans lesquels on propose parfois des méthodes permettant le ralentissement du vieillissement. Ces ouvrages alimentent la médiatisation du thème «santé, sport et vieillissement » valorisant l'idéologie du corps jeune, beau, élancé, produit d'une vie saine et sportive.

Dans cette perspective, la sécurité devient alors pour les seniors une question cruciale. Ils semblent souvent partagés entre le désir de faire beaucoup de sport pour se maintenir en forme et la réalité des blessures occasionnées par une pratique trop intensive. Cette ambiguïté permet de mieux comprendre leurs nouvelles pratiques: baisse de la multipratique et augmentation de la fréquence des monopratiques. Cette question de la sécurité revêt toujours plus d'acuité avec l'âge et s'exprime par une réduction progressive de la prise de risque et de la recherche de sensations. S'engager dans une nouvelle activité ou pratiquer occasionnellement devient alors un facteur de risque car le corps n'est pas préparé. Pour la même raison, les seniors se désengagent massivement de la compétition. La performance n'est plus une motivation prioritaire. Mais ce basculement n'est pas immédiat, et c'est un phénomène complexe qui mêle d'une part le souhait de combattre le vieillissement, d'afficher une image jeune et d'autre part la réalité d'un corps qui vieillit, qu'il faut entretenir et surtout préserver. Raymonde Feillet (2000) analyse ce mécanisme de résistance et d'acceptation du corps vieillissant, qui conduit à la fois à rejeter les pratiques engagées car elles font prendre conscience de la fragilité du corps (problèmes de santé, blessures, etc.) et les pratiques trop douces car elles signifient la vieillesse, la perte de capacité et la sortie du monde des actifs. Le choix des activités est le résultat d'une décision articulée entre le besoin de «vivre pleinement sa retraite » et le risque physique réel lié à la pratique (Feillet, 2006). Ainsi, l'engagement physique demeure encore une motivation importante, y compris chez $86 \%$ des 70-75 ans. De même, le regain d'intérêt 
des 65-70 ans pour la performance sportive pourrait illustrer ce phénomène de résistance au vieillissement. La question demeure ouverte. Le maintien de l'engagement dans des compétitions auxquelles participent encore des actifs est typique de ce comportement. L'exigence de mesurer sa force dans des activités physiques comprend celle de se surpasser, de « se sentir fatigué ». Il est évident que ces analyses renvoient à des schémas généraux qui ne doivent pas occulter la pluralité des formes de pratique en fonction des trajectoires et des caractéristiques sociales.

Comme le montrent les activités préférées des seniors, deux types de pratique semblent satisfaire à cette double exigence de santé et de sécurité. D'une part des exercices d'intérieur orientés vers l'entretien du corps et d'autre part des activités de plein air. Le contact avec la nature est ainsi une motivation importante à partir de 50 ans (tableau 14, page 152). Derrière ce phénomène se profile également une certaine philosophie de vie, une relation à l'environnement plus paisible et moins conflictuelle. La pratique sportive, plus ou moins exigeante, sert alors de prétexte à la découverte de nouveaux espaces naturels. Le développement particulièrement soutenu de la randonnée en montagne chez les seniors (Burlot, Lefèvre, 2004), comme de toutes les formes de marche, atteste ce phénomène.

En revanche, et contrairement à de nombreuses hypothèses, les seniors sont loin de considérer le sport comme un moyen privilégié de sociabilité. Certes, la rencontre avec les autres et le plaisir d'être avec des proches restent de sérieuses motivations, mais on constate une baisse progressive de l'intérêt porté par les seniors à ces questions, qui s'accélère chez les plus de 70 ans. Cette tendance confirme le phénomène de désengagement institutionnel souligné plus haut, ce qui ne signifie évidemment pas que les seniors ne recherchent plus aucune sociabilité. Si la sociabilité sportive n'est pas le ressort le plus important pour les seniors, elle n'en demeure pas moins un facteur essentiel de fidélisation au club ou de participation aux cours, dont le sportif n'est pas toujours conscient. Les études menées (Lefèvre, Burlot, 2004; De Bruyn, 2006) sur les processus d'abandon montrent que les relations sociales et la convivialité jouent un rôle déterminant dans la fidélisation des pratiquants et a contrario dans les processus d'abandon: «On vient pour la pratique sportive mais on reste pour les copains ».

\section{Conclusion}

Même si l'on constate une diminution des pratiques à 50 ans, les activités physiques et sportives des seniors s'inscrivent dans une forme de continuité par rapport à celles des plus jeunes. D'un côté la non- pratique continue de progresser après 50 ans parmi les non-retraités. D'un autre côté, même si des interrogations demeurent sur les mouvements internes à cette population, la part des sportifs chez les retraités semble se stabiliser. Le passage à la retraite ne paraît pas constituer une phase de conversion radicale des non-sportifs au sport, même si les seniors sportifs deviennent très assidus au moment de la retraite. Les taux de pratique demeurent à peu près constants entre 50 et 70 ans. L'hypothèse selon laquelle, en ce début de XXIe siècle, les seniors allaient occuper massivement leur temps libre avec des pratiques sportives doit donc être formulée avec circonspection. La question est complexe et mérite des analyses que ne permet pas la photographie instantanée d'une enquête. De plus, le passage à la retraite s'accompagne à la fois d'un sentiment de vouloir la vivre pleinement et d'une espèce de gêne, voire de «mal-être » face à cette nouvelle situation « d'inactif ». Lors de cette transition se mélangent d'une part la volonté de continuer à pratiquer le sport comme les plus jeunes et d'autre part la prise de conscience d'un corps vieillissant à ménager. Les pratiques sportives sont paradoxales car elles sont à la fois synonymes de résistance et de révélateur $\mathrm{du}$ vieillissement. Le sport chez les seniors s'inscrit dans une certaine forme de continuité et de stabilité, révélant une réelle diversité selon le sexe et le positionnement économique et culturel. 
Parallèlement, les pratiques subissent un certain nombre de changements en partie provoqués par le vieillissement. Les motivations des seniors sont liées à la santé à la sécurité et se traduisent par l'éventail de leurs pratiques, leurs activités privilégies et les modalités de l'engagement. Ainsi les seniors se risquent moins à la nouveauté, ont des 154 pratiques moins institutionnalisées, et se concentrent progressivement sur une seule activité, mais avec une fréquence plus élevée. Ils se détournent progressivement des pratiques à forte dépense physique et à risque comme les sports collectifs. Ils se consacrent plus volontiers à des exercices moins exigeants, centrés sur le corps et le bien-être, à l'image des gymnastiques douces ou d'entretien. Si de 50 à 69 ans, il ne semble s'opérer que des redéfinitions de modalités de pratique, l'implication des 70 à 75 ans se caractérise par une véritable rupture qualitative et quantitative. Les taux de pratique se réduisent considérablement, confirmant cette frontière entre le «troisième âge », celui de la retraite active et un " quatrième âge » synonyme de vieillesse.

\section{Bibliographie}

BARTHE J.-F., CLÉMENT S., DRULHE M., 1988, « Vieillesse ou vieillissement. Les processus d'organisation des modes de vie chez les personnes âgées », Les Cahiers de la recherche sur le travail social, $\mathrm{n}^{\circ} 15, \mathrm{p} .11-31$.

BAREllo A., ABBÀ A., PERALMA L., FUBUNi E., 2002, « Activité physique chez les personnes âgées: résultats d'une enquête menée sur un échantillon d'hommes et de femmes de plus de 50 ans, retraités et vivant en Italie du Nord ou dans le centre », Biométrie humaine et anthropologie, $n^{\circ} 3-4$, p. 227-234.

BOUCHET P., 2002, «Une nouvelle tendance de consommation chez les personnes âgées: le tourisme sportif haut de gamme », Loisir et Société, n²5, p. 377-396.

CARADEC V., 2003, « Comportements culturels de la population âgée », Empan, n 52, p. 54-61.

CARADEC V., 2001, Sociologie de la vieillesse et du vieillissement, Paris, Nathan.

CARADEC V., 2004, Vieillir après la retraite. Approche sociologique du vieillissement, Paris, PUF.

CIBOIS P., 1993, « Le PEM, pourcentage de l'écart maximum : un indice de liaison entre modalités d'un tableau de contingence », Bulletin de méthodologie sociologique, n 40, p. 43-63.

CLÉMENT S., 2003, « Le vieillissement avec le temps et malgré le temps », Empan, n 52, p. 1422.

CROSNIER D., 2005, «Les activités physiques et sportives des seniors » in Muller L. (coord.), La pratique des activités physiques et sportives en France, Paris, Ministère de la Jeunesse, des Sports et de la Vie associative et Insep, p. 81-98.

CUMMING E. HENRY W., 1961, Growing Old. The process of disengagement, New-York, Basic Books.

DE BRUYN F., 2006, Biographies et carrières plurielles: analyses des interactions entre la carrière sportive des nageurs et les autres domaines de la vie sociale, thèse de Doctorat, Université de Nanterre — Paris.

DEJARDIN P., BIGOT J., DROLEZ B., DEL Rio F., 1999, « L'équilibre et le senior », Centre d'informations sociales et d'actions gérontologiques, Troyes. 
DESPLANQUES G., 2005, «La population française âgée », Retraite et société, $\mathrm{n}^{\circ}$ 45, Paris, La Documentation française, p. 10-23.

DESSAINT M.-P., 2005, Une retraite heureuse? Ça dépend de vous! Québec, Flammarion.

DONNAT 0., 1998, Les pratiques culturelles des français, enquête 1997, Paris, La Documentation française.

DIRN L, 1998, La société française en tendance, 1975-1995, Paris, PUF.

ERRAIS B., IRLINGER P. (COORD.), LOUVEAU C., METOUDI M., POCCIELLO C., 1984, Les usages sportifs du temps libéré; recherche et analyse des facteurs de développement - Étude de faisabilité d'une enquête nationale, Paris, Insep.

FAUROBERT L., 1976, Culture physique du troisième âge, Paris, Amphora.

FEILLET R., 2000, Pratiques sportives et résistance au vieillissement, Paris, L'Harmattan.

FEILLET R., RONCIN C. (DIR.), 2006, Souci du corps, sport et vieillissement, Ramonville SaintAgne, Erès.

FOLLEA L., 22 AVRIL 1997, « La consommation des seniors est appelée à se développer fortement », Le Monde.

GAULLIER X., 1993, «Avenir à reculons, Bilan démographique 1993 — projection démographiques à l'horizon 2050 », Liaison sociales, n 41/93.

GAULLIER X., 2003, Le temps des retraites. Les mutations de la société salariale, Paris, Le Seuil.

HENAFF-PINEAU R-C., 2008, Pratiques physiques des seniors et vieillissement: entre raison et passion. Analyse sociologique de la transformation des pratiques avec l'avancée en âge, thèse de Doctorat en Sciences du Sport, de la Motricité et du Mouvement Humain, Université Paris-Sud 11.

LEFÈVRE B., BURLOT F., 2004, Analyse démographique et sociologique des licenciés de la Fédération Française de Montagne et d'Escalade, rapport de recherche n'Insep/FFME.

LONGUEVILLE L., 1978, L'art et les manières du mieux vieillir, Paris, Le Centurion.

LORIAUX M., 2004, « Production, consommation et développement sociétal », Gérontologie, n 132, p. 18-23.

LOUVEAU C., 2002, «Enquêter sur les pratique sportives des Français: tendances lourdes et problèmes méthodologiques. » in Mignon P., Truchot G., Les pratiques sportives en France, lnsep, p. 135-154.

IRLINGER P. (RESPONSABLE), LOUVEAU C., METOUDI M., 1987, Rapport de recherche, les pratiques sportives des français; usages sportifs du temps libéré, Paris.

INSEE-MJS-MINISTÈRE DE LA CULTURE, 2005, La pratique des activités physiques et sportives en France, lnsep.

INSEE, 1990, «Les personnes âgées de 60 ans et plus en France métropolitaine par régions en $1990,2000,2010,2020 »$ in recensement. 
MIGNON P, TRUCHOT G., 2002, Les pratiques sportives en France. Enquête 2000, Ministère des Sports et lnsep.

MIGNON P, TRUCHOT G., « Entre 2000 et 2003, la pratique a-t-elle progressé ? » in La pratique des activités physiques et sportives en France, p. 181-196.

PARANT A., 1998, «L'avenir incertain des seniors », Futuribles, n² 233, p. 65-72.

PICHOT L., 2002, «Les expériences de loisirs sportifs chez les personnes de plus de 50 ans:

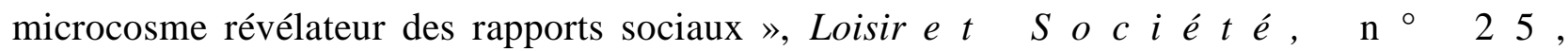
p $329-347$

PINSON G., 2006, « La population de la France en 2005 », Population et Sociétés, n 421.

POCIELLO C., 1999, Sport et sciences sociales, Paris, Vigot, collection Repères.

RICHET-MASTAIN L., 2006, «Bilan démographique 2005. En France, la fécondité des femmes augmente toujours », Insee première, $\mathrm{n}^{\circ} 1059$.

ROCHEFORT R., 2000, Vive le papy-boom, Paris, Odile Jacob.

SARRE V., 2002, «Les vétérans font durer le plaisir », En Jeu, une autre idée du sport, n 355. p. $10-14$.

TRUCHOT G., 2002, «Les seniors » in Mignon Patrick, Guy Truchot, Les pratiques sportives en France. Enquête 2000, Ministère des Sports et Insep.

\section{Notes :}

1 Dans cette présentation, nous préférerons le terme « seniors » aux expressions « troisième âge » ou « personnes âgées » pour identifier notre population.

2 Voir sur cette question la controverse historique entre l'Insee et l'Insep (Pociello, 1999).

3 Q2bis: «Afin de préciser et pour être bien sûr de ne rien avoir oublié, je vais vous citer une liste d'activités. Y a-t-il des activités que vous avez pratiquées au cours des 12 derniers mois et que vous n'avez pas citées?».

4 Selon les contraintes des analyses, le découpage de l'âge pourra cependant être différent.

5 Ce pic n'apparait pas dans le Stat Info n ${ }^{\circ} 01 / 01$ en raison de l'aspect approximatif de la courbe, ni dans l'ouvrage sur les pratiques sportives des Français (Mignon, Truchot, 2002) qui analyse les seniors à partir de 55 ans.

6 Pour les moins riches, on devrait davantage parler d'un sursaut de non-pratique pour les 60-64 ans.

\section{Résumé :}

Cet article a pour objet les pratiques physiques et sportives des seniors. Il se fonde sur un traitement secondaire de l'enquête 2000 sur les pratiques sportives des Français, dernière enquête nationale à appréhender le phénomène de la pratique sportive dans sa diversité. Il se propose d'analyser les spécificités de la population senior sur le plan de l'investissement sportif et des modalités de pratique privilégiées. D'une façon générale, le choix d'analyser les seniors à partir de 50 ans trouve une réelle pertinence. L'analyse des données met en évidence une rupture non négligeable de 
l'engagement sportif sur la tranche d'âge 50-54 ans. Cette rupture ne doit cependant pas masquer d'une part, un effritement régulier de l'engagement sportif avec l'âge: dans la continuité des moins de 50 ans, la part des sportifs parmi les non retraités après 50 ans est en effet de moins en moins importante alors qu'elle est stable chez les retraités; et d'autre part, des disparités à l'intérieur des seniors en fonction du sexe, de la retraite, de la richesse et des diplômes.

Concernant les modalités de pratiques, les seniors ne se risquent plus à essayer de nouvelles pratiques et se recentrent progressivement sur une seule activité. Ils se désengagent progressivement des pratiques à fort engagement physique et à risque, comme les sports collectifs, et s'engagent plutôt dans des activités modérées. Si pour les 50-69 ans, il ne semble s'opérer que des redéfinitions de modalités de pratique, il existe une véritable rupture en termes d'engagement sportif pour les 7075 ans, les taux de pratique se réduisant considérablement. 